Boise State University

ScholarWorks

Mathematics Faculty Publications and

Presentations

Department of Mathematics

4-2016

Decompositions of Ideals of Minors Meeting a Submatrix

Kent M. Neuerburg

Southeastern Louisiana University

Zach Teitler

Boise State University

This is an Accepted Manuscript of an Article published in Communications in Algebra (2016), available online at doi: 10.1080/00927872.2015.1027375 


\title{
DECOMPOSITIONS OF IDEALS OF MINORS MEETING A SUBMATRIX
}

\author{
KENT M. NEUERBURG AND ZACH TEITLER
}

\begin{abstract}
We compute the primary decomposition of certain ideals generated by subsets of minors in a generic matrix or in a generic symmetric matrix, or subsets of Pfaffians in a generic skew-symmetric matrix. Specifically, the ideals we consider are generated by minors that have at least some given number of rows and columns in certain submatrices.
\end{abstract}

\section{INTRODUCTION}

The paper [1] concerns ideals of minors fixing a submatrix, meaning the set of minors in an $m \times n$ matrix that involve all $r$ of the first $r$ columns of the matrix. One of the main results of that paper, Theorem A, gives the primary decomposition of the ideal generated by this set of minors. We generalize this to consider minors that involve at least $r$ of the first a columns:

Theorem 1.1. Let $k$ be a field, let $X$ be a generic $m \times n$ matrix, that is $X=\left(x_{i, j}\right)$ for $1 \leq i \leq m, 1 \leq j \leq n$, and let $R=k[X]=k\left[x_{i, j}\right]$. Regard $X$ as a block matrix, $X=(A B)$, where $A$ has size $m \times a$ and $B$ has size $m \times(n-a)$. Let $J$ be the ideal generated by the set of t-minors of $X$ that involve at least $r$ columns of $A$, let $I_{t}(X)$ be the ideal generated by the t-minors of $X$, and similarly let $I_{r}(A)$ be the ideal generated by the r-minors of $A$. Then $J=I_{t}(X) \cap I_{r}(A)$.

We generalize further than this, to allow several blocks as well as restrictions on both rows and columns. We also give similar statements for ideals generated by sets of minors of a generic symmetric matrix, requiring some number of rows or columns in certain submatrices. Before we give these statements, we consider one possible application in the setting of the two-block theorem above.

It is sometimes useful to consider, for a homogeneous ideal $I$, the ideal $I_{\leq d}$ generated by the forms in $I$ of degree $\leq d$. For example, in resolving the singularities of the affine cone $V(I) \subset \mathbb{A}^{n}$, upon blowing up the origin, the total transform of $I$ may have embedded components supported along the projective variety $V\left(I_{\leq d}\right)$, lying in the exceptional divisor $\cong \mathbb{P}^{n-1}$, for various $d$; see [10]. When $I$ is a determinantal ideal, generated by minors of a matrix whose entries are homogeneous forms, then $I_{\leq d}$ is generated by just some of the minors of the matrix.

Corollary 1.2. Let $X=\left(x_{i, j}\right)$ be a generic $m \times n$ matrix, regarded as consisting of two blocks, $X=(A B)$, where $A$ has size $m \times a$ and $B$ has size $m \times(n-a)$. Fix the ring

Date: June 24, 2014.

2010 Mathematics Subject Classification. 13F50, 13C40.

Key words and phrases. Determinantal ideals, Pfaffian ideals, primary decomposition, straightening laws.

The authors were partially supported by a grant from the University of Louisiana Board of Regents, LEQSF(2007-10)-RD-A-28. 
$R=k[X]=k\left[x_{i, j}\right]$ where every entry in $A$ has degree $p$ and every entry in $B$ has degree $q>p$; that is, $\operatorname{deg}\left(x_{i, j}\right)=p$ if $1 \leq j \leq a, \operatorname{deg}\left(x_{i, j}\right)=q$ otherwise. Fix $t$ and $d$. Let $I_{t}(X)_{\leq d}$ be the ideal generated by those $t$-minors of $X$ of degree less than or equal to $d$, and for each $r$ let $I_{r}(A)$ be the ideal generated by the r-minors of $A$. Then $I_{t}(X)_{\leq d}=I_{t}(X) \cap I_{r}(A)$ for $r=\left\lceil\frac{t q-d}{q-p}\right\rceil$.

Indeed, a $t \times t$ minor $M$ with $r$ columns in $A$ and $t-r$ columns in $B$ will have degree $\operatorname{deg} M=p r+q(t-r)$; the value of $r$ in the statement is the least integral solution to $\operatorname{deg} M \leq d$.

Corollary 1.3. Consider the vector bundles $F=\mathcal{O}_{\mathbb{P}^{N}}^{m}, G=\mathcal{O}_{\mathbb{P}^{N}}(p)^{\oplus a} \oplus \mathcal{O}_{\mathbb{P}^{N}}(q)^{\oplus n-a}$. Let $f: F \rightarrow G$ be a general map, let $f^{\prime}: F \rightarrow \mathcal{O}_{\mathbb{P}^{N}}(p)^{\oplus a}$ be the induced map, let $\Delta_{t}(f)$ be the degeneracy locus, $\Delta_{t}(f)=\left\{x \in \mathbb{P}^{N} \mid \operatorname{rank} f_{x}<t\right\}$, and let $\Delta_{t}(f)_{\leq d}$ be the locus defined by the ideal $I\left(\Delta_{t}(f)\right)_{\leq d}$. Then $\Delta_{t}(f)_{\leq d}=\Delta_{t}(f) \cup \Delta_{r}\left(f^{\prime}\right)$ for $r=\left\lceil\frac{t q-d}{q-p}\right\rceil$.

This is similar to [11], which dealt with $\mathbb{P}^{2}$ and had $n=m+1$ in order to obtain general Hilbert-Burch matrices of a given type. (In particular [11, Prop. 3.4] simply recreated a special case of $[1$, Thm. A].)

Sections 2 and 3 review some background of posets, dosets, algebras with straightening law, and doset algebras with straightening law. Then we give our results for minors in generic matrices (Section 4), minors in generic symmetric matrices (Section 5), and Pfaffians in generic skew-symmetric matrices (Section 6).

Throughout, all rings are commutative with unity.

\section{Orders AND Straightening}

A poset (partially ordered set) is a set together with a transitive, reflexive, antisymmetric relation $\leq$.

Definition 2.1 ([2, Definition 1.0.3]). A doset of a poset $P$ is a subset $D \subset P \times P$ such that

(1) $(a, a) \in D$ for all $a \in P$,

(2) if $(a, b) \in D$ then $a \leq b$, and

(3) if $a \leq b \leq c \in P$, then $(a, c) \in D$ if and only if $(a, b) \in D$ and $(b, c) \in D$.

Example 2.2. (1) Let $[n]=\{1, \ldots, n\}$ and let $P_{n}=2^{[n]}$, the power set of $[n]$. We order $P_{n}$ as follows. For $A=\left\{a_{1}<\cdots<a_{s}\right\} \subset[n]$ and $B=\left\{b_{1}<\cdots<b_{t}\right\} \subset[n], A \leq B$ if and only if $s \geq t$ and $a_{i} \leq b_{i}$ for $i=1, \ldots, t$. This makes $P_{n}$ a poset.

Note, $A \leq B$ if and only if in the diagram

\begin{tabular}{|l|l|l|l|l|l|}
\hline$a_{1}$ & $a_{2}$ & $\cdots$ & $a_{t}$ & $\cdots$ & $a_{s}$ \\
\hline$b_{1}$ & $b_{2}$ & $\cdots$ & $b_{t}$ & \multicolumn{1}{|c}{} \\
\cline { 1 - 3 }
\end{tabular}

the first row is at least as long as the second and the entries are weakly increasing down each column.

(2) Fix $m$ and $n$. Let $P_{m, n} \subset P_{m} \times P_{n}$ consist of pairs of $\operatorname{subsets}(A, B)$ such that $|A|=|B|$, with $(A, B) \leq\left(A^{\prime}, B^{\prime}\right)$ if and only if $A \leq A^{\prime}$ and $B \leq B^{\prime}$.

(3) Let $D_{n} \subset P_{n} \times P_{n}$ consist of pairs $(A, B)$ such that $|A|=|B|$ and $A \leq B$. Then $D_{n}$ is a doset.

Example 2.3. Here are some key examples of posets and dosets of minors in matrices. 
(1) $P_{m, n}$ is the poset of minors (of an $m \times n$ matrix): $(A, B) \in P_{m, n}$ corresponds to the minor with rows indexed by $A$ and columns indexed by $B$. This poset is usually denoted $\Delta(X)$, where $X$ is an $m \times n$ matrix.

(2) $D_{n}$ is the doset of minors of a symmetric $n \times n$ matrix: $(A, B) \in D_{n}$ corresponds to the minor with rows indexed by $A$ and columns indexed by $B$. An element of $D_{n}$ is called a doset minor. We denote this poset $\Delta^{s}(Y)$, where $Y$ is a symmetric $n \times n$ matrix. (It is denoted $\Delta(Y)$ in [2]; we adjoin the $s$ for "symmetric" in order to avoid ambiguity.)

The condition $A \leq B$ means that a minor is a doset minor if and only if the main diagonal of the minor lies in the upper triangle of the matrix (including the diagonal).

(3) Let $P_{n}(2)$ be the subset of $A \in P_{n}$ such that $|A|$ is even. Then $P_{n}(2)$ is the poset of Pfaffians of a skew-symmetric $n \times n$ matrix: $A \in P_{n}(2)$ corresponds to the Pfaffian of the submatrix with rows and columns indexed by $A$. Following [2] we denote this poset $\Pi(Z)$, where $Z$ is a skew-symmetric $n \times n$ matrix.

Definition 2.4 ([3, §4.A], [2, Definition 1.0.1]). Let $A$ be a $B$-algebra and $P \subset A$ a subset with a partial order $\leq$. Then $A$ is a graded algebra with straightening law (abbreviated ASL) on $P$ over $B$ if

(1) $A=\bigoplus_{i \geq 0} A_{i}$ is a graded $B$-algebra such that $A_{0}=B, P$ consists of homogeneous elements of positive degree, and $P$ generates $A$ as a $B$-algebra.

(2) $A$ is a free $B$-module with a basis given by products $\xi_{1} \cdots \xi_{m}, m \geq 0, \xi_{i} \in P$, such that $\xi_{1} \leq \cdots \leq \xi_{m}$. These products are called standard monomials.

(3) For all incomparable $\xi, \nu \in P$, the product $\xi \nu$ can be written as a combination of standard monomials

$$
\xi \nu=\sum a_{\mu} \mu, \quad a_{\mu} \in B, a_{\mu} \neq 0, \quad \mu \text { standard monomial, }
$$

in which every $\mu$ contains a factor $\zeta \in P$ such that $\zeta \leq \xi$ and $\zeta \leq \nu$. These are called straightening relations.

Definition 2.5 ([2, Definition 1.0.4]). Let $A$ be a $B$-algebra and $D \subset A$ a subset such that $D$ is a doset of a poset $P$. Then $A$ is a graded doset algebra with straightening law (abbreviated DASL) on $D$ over $B$ if

(1) $A=\bigoplus_{i \geq 0} A_{i}$ is a graded $B$-algebra such that $A_{0}=B, D$ consists of homogeneous elements of positive degree, and $D$ generates $A$ as a $B$-algebra.

(2) $A$ is a free $B$-module with a basis given by products $\left(\alpha_{1}, \alpha_{2}\right) \cdots\left(\alpha_{2 k-1}, \alpha_{2 k}\right), k \geq 1$, $\left(\alpha_{2 i-1}, \alpha_{2 i}\right) \in D, \alpha_{1} \leq \cdots \leq \alpha_{2 k}$. These products are called standard monomials.

(3) Suppose $M=\left(\alpha_{1}, \alpha_{2}\right) \cdots\left(\alpha_{2 k-1}, \alpha_{2 k}\right)$, with standard representation $M=\sum \lambda_{N} N$, $0 \neq \lambda_{N} \in B$, each $N$ a standard monomial. Let $N=\left(\beta_{1}, \beta_{2}\right) \cdots\left(\beta_{2 \ell-1}, \beta_{2 \ell}\right)$ be one of the standard monomials appearing in the standard representation of $M$. Then for every permutation $\sigma$ of $\{1, \ldots, 2 k\}$, the sequence $\left\{\alpha_{\sigma(1)}, \ldots, \alpha_{\sigma(2 k)}\right\}$ is lexicographically greater than or equal to the sequence $\left(\beta_{1}, \ldots, \beta_{2 \ell}\right)$.

(4) In the notation above, if there is a permutation $\sigma$ such that $\alpha_{\sigma(1)} \leq \cdots \leq \alpha_{\sigma(2 k)}$ then the standard monomial $\left(\alpha_{\sigma(1)}, \alpha_{\sigma(2)}\right) \cdots\left(\alpha_{\sigma(2 k-1)}, \alpha_{\sigma(2 k)}\right)$ must appear in the standard representation of $M$ with coefficient \pm 1 .

Example 2.6. Fix an arbitrary commutative $\operatorname{ring} B$ with unity. 
(1) Let $X$ be an $m \times n$ generic matrix, that is $X=\left(x_{i, j}\right), 1 \leq i \leq m, 1 \leq j \leq n$, the $x_{i, j}$ variables over $B$. Then $A=B[X]=B\left[x_{i, j}\right]$ is a graded ASL on $\Delta(X)$ over $B[3$, Chap. 4],[2, Thm. 1.0.5].

(2) Let $Y$ be an $n \times n$ generic symmetric matrix, that is $Y=\left(y_{i, j}\right), 1 \leq i, j \leq n$, $y_{i, j}=y_{j, i}$. Then $A=B[Y]$ is a graded DASL on $\Delta^{s}(Y)$ over $B$ [2, Thm. 1.0.10].

(3) Let $Z$ be an $n \times n$ generic skew-symmetric matrix, that is $Z=\left(z_{i, j}\right), 1 \leq i, j \leq n$, $z_{i, j}=-z_{j, i}, z_{i, i}=0$. Then $A=B[Z]$ is a graded ASL on $\Pi(Z)$ over $B$ [2, Thm. 1.0.14].

\section{ORDER IDEALS}

We use ASLs and DASLs entirely for the following properties.

Definition 3.1. Let $P$ be a poset. An order ideal is a subset $I \subset P$ such that if $\alpha \in I$ and $\beta \leq \alpha$ then $\beta \in I$. The order ideal generated by $S \subset P$ is the smallest order ideal containing $S$, that is, $\{\alpha \in P \mid \alpha \leq s$ for some $s \in S\}$. The order ideal cogenerated by $S \subset P$ is the largest order ideal disjoint from $S$, that is, $\{\alpha \in P \mid \alpha \not \leq s$ for all $s \in S\}$.

When $A$ is an ASL on $P$ and $I \subset P$, we write $A I$ for the (ring) ideal generated by $I$.

Lemma 3.2 ([3, Prop. 5.2]). Let $A$ be an $A S L$ on $P$ and let $I, J \subset P$ be ideals. Then $A I \cap A J=A(I \cap J)$.

We will prove a similar lemma for DASLs. First, we introduce a partial order for dosets.

Definition 3.3. Let $D$ be a doset of $P$. Then $D$ is a poset with the partial order $(a, b) \leq_{1}$ $(c, d)$ if and only if $a \leq c$ in $P$. A doset order ideal is an order ideal in the poset $\left(D, \leq_{1}\right)$. As before, the ideal generated by $S \subset D$ is the smallest ideal containing $S$ and the ideal cogenerated by $S \subset D$ is the largest ideal disjoint from $S$.

A DASL on $D$ is not necessarily an ASL on $\left(D, \leq_{1}\right)$. Again when $A$ is a DASL on $D$ and $I \subset D$ is a doset order ideal, we write $A I$ for the ring ideal generated by $I$.

Lemma 3.4. Let $A$ be a DASL on $D$ over $B$ and let $I \subset D$ be a doset order ideal. Then $A I$ is spanned over $B$ by the standard monomials $N=\left(\beta_{1}, \beta_{2}\right) \cdots\left(\beta_{2 \ell-1}, \beta_{2 \ell}\right)$ such that $\left(\beta_{1}, \beta_{2}\right) \in I$.

Proof. Let $\left(\alpha_{1}, \alpha_{2}\right) \in I, f \in A$, and let $N=\left(\beta_{1}, \beta_{2}\right) \cdots\left(\beta_{2 \ell-1}, \beta_{2 \ell}\right)$ be one of the standard monomials appearing in the standard representation of $\left(\alpha_{1}, \alpha_{2}\right) f$. The sequence $\left(\beta_{1}, \beta_{2}, \ldots, \beta_{2 \ell}\right)$ is lexicographically less than or equal to $\left(\alpha_{1}, \alpha_{2}\right)$, so in particular $\beta_{1} \leq \alpha_{1}$. Hence $\left(\beta_{1}, \beta_{2}\right) \leq_{1}$ $\left(\alpha_{1}, \alpha_{2}\right)$ and hence $\left(\beta_{1}, \beta_{2}\right) \in I$. Thus every standard monomial appearing in every element of $A I$ has a factor in $I$.

Lemma 3.5. Let $A$ be a $D A S L$ on $D$ and let $I, J \subset D$ be ideals. Then $A I \cap A J=A(I \cap J)$.

Proof. A standard monomial $N=\left(\beta_{1}, \beta_{2}\right) \cdots\left(\beta_{2 \ell-1}, \beta_{2 \ell}\right)$ appearing in the standard representation of an element of $A I \cap A J$ has $\left(\beta_{1}, \beta_{2}\right) \in I$ and $\in J$, hence in $I \cap J$. This shows $A I \cap A J \subset A(I \cap J)$ and the reverse inclusion is obvious.

Finally we recall the following results.

Proposition 3.6 ([3, Thm. 6.3]). Let $B$ be a domain, $X$ a generic matrix, $A=B[X]$, and $\delta \in \Delta(X)$, the poset of minors (see Example 2.3(1)). Let $I(X, \delta)$ be the ideal in $A$ generated by the order ideal cogenerated by $\delta$. Then $I(X, \delta)$ is a prime ideal. 
This is an author-produced, peer-reviewed version of this article. The final, definitive version of this document can be found online at Communications in Algebra, published by Taylor and Francis. Copyright restrictions may apply. doi: 10.1080/00927872.2015.1027375

DECOMPOSITIONS OF IDEALS OF MINORS MEETING A SUBMATRIX

Proposition 3.7 ([8, Theorem 1], [5, Remark 2.5(a)]). Let B be a domain, $Y$ a generic symmetric matrix, $A=B[Y]$, and $\delta \in \Delta^{s}(Y)$, the doset of minors (see Example 2.3(2)). Let $I(Y, \delta)$ be the ideal in $A$ generated by the doset order ideal cogenerated by $\delta$. Then $I(Y, \delta)$ is a prime ideal.

Proposition 3.8 ([2, Thm. 2.1.12]). Let $B$ be a domain, $Z$ a generic skew-symmetric matrix, $A=B[Z]$, and $\delta \in \Pi(Z)$, the poset of Pfaffians (see Example 2.3(3)). Let $I(Z, \delta)$ be the ideal in $A$ generated by the order ideal cogenerated by $\delta$. Then $I(Z, \delta)$ is a prime ideal.

\section{MinORS}

We are interested in ideals generated by certain sets of $t$-minors in a generic matrix $X$. Specifically, we will require the generating minors to have at least $r_{1}$ rows in the first $R_{1}$ rows of $X$, at least $r_{2}$ rows contained in the first $R_{2}$ rows of $X$, and so on; and similarly for columns.

Let $X$ be a generic $m \times n$ matrix, $X=\left(x_{i, j}\right)$ for $1 \leq i \leq m, 1 \leq j \leq n$, and fix $A=B[X]=B\left[\left\{x_{i, j}\right\}\right]$ for a commutative $\operatorname{ring} B$ with unity. For $1 \leq t \leq \min (m, n)$, a $t$-minor may be specified by listing its rows and columns; we write $\left[a_{1}, \ldots, a_{t} \mid b_{1}, \ldots, b_{t}\right]$, where $1 \leq a_{1}<\cdots<a_{t} \leq m$ and $1 \leq b_{1}<\cdots<b_{t} \leq n$, for the minor with rows $a_{1}, \ldots, a_{t}$ and columns $b_{1}, \ldots, b_{t}$.

Fix sequences $1 \leq R_{1} \leq \cdots \leq R_{p} \leq m$ and $1 \leq C_{1} \leq \cdots \leq C_{q} \leq n$ where $p, q \geq 0$. The sequences $R=\left(R_{1}, \ldots, R_{p}\right)$ and $C=\left(C_{1}, \ldots, C_{q}\right)$ (possibly empty if $p=0$ or $q=0$ ) describe the division of $X$ into row and column blocks, respectively. Specifically, let $X_{R_{i}}$ be the submatrix of $X$ consisting of the first $R_{i}$ rows and let $X^{C_{j}}$ be the submatrix consisting of the first $C_{j}$ columns. Fix also sequences $r=\left(r_{1}, \ldots, r_{p}\right)$ and $c=\left(c_{1}, \ldots, c_{q}\right)$.

We are interested in the $t$-minors that have at least $r_{i}$ rows contained in $X_{R_{i}}$ and at least $c_{j}$ columns contained in $X^{C_{j}}$, for each $i, j$ (with no restriction if $p=0$ or $q=0$ ).

Theorem 4.1. Let $B$ be a ring and $A=B[X]$. Let $J=J(X, t, R, C, r, c)$ be the ideal generated by t-minors of $X$ that have at least $r_{i}$ rows contained in $X_{R_{i}}$ for each $1 \leq i \leq p$ (no restriction if $p=0$ ) and at least $c_{j}$ columns contained in $X^{C_{j}}$ for each $1 \leq j \leq q$ (no restriction if $q=0)$. Then

$$
J=I_{t}(X) \cap I_{r_{1}}\left(X_{R_{1}}\right) \cap \cdots \cap I_{r_{p}}\left(X_{R_{p}}\right) \cap I_{c_{1}}\left(X^{C_{1}}\right) \cap \cdots \cap I_{c_{q}}\left(X^{C_{q}}\right) .
$$

Example 4.2. When $p=q=0, J=I_{t}(X)$.

When $p=0$ and $q=1$, we are in the two-block setting of the Introduction. If also $c_{1}=C_{1}$, we recover [1, Thm. A].

Remark 4.3. We are essentially working with the special case of Mohammadi's block adjacent simplicial complexes [9] in which each block is contained in the previous one and they all have the last column of the matrix as a common endpoint (in Mohammadi's indexing; for us, we take blocks to start at the first column or row). Unlike Mohammadi, we allow non-maximal minors, we allow restrictions on both the rows and columns appearing in the minor, and we allow the overlaps between "consecutive" blocks to be arbitrarily large.

Proof. Each of the following sets of minors is an order ideal in $\Delta(X)$ :

(1) The set of minors of size $\geq t$, the generating set of $I_{t}(X)$, is the order ideal generated by $[m-t+1, \ldots, m \mid n-t+1, \ldots, n]$, or cogenerated by $[1, \ldots, t-1 \mid 1, \ldots, t-1]$.

(2) The set of $\left(\geq r_{i}\right)$-minors of $X_{R_{i}}$ is the order ideal generated by $\left[R_{i}-r_{i}+1, \ldots, R_{i} \mid\right.$ $\left.n-r_{i}+1, \ldots, n\right]$, or cogenerated by $\left[1, \ldots, r_{i}-1, R_{i}+1, \ldots, n \mid 1, \ldots, n-R_{i}+r_{i}-1\right]$. 
This is an author-produced, peer-reviewed version of this article. The final, definitive version of this document can be found online at Communications in Algebra, published by Taylor and Francis. Copyright restrictions may apply. doi: 10.1080/00927872.2015.1027375

(3) Similarly, the set of $\left(\geq c_{j}\right)$-minors of $X^{C_{j}}$ is the order ideal generated by $[m-$ $\left.c_{j}+1, \ldots, m \mid C_{j}-c_{j}+1, \ldots, C_{j}\right]$, or cogenerated by $\left[1, \ldots, n-C_{j}+c_{j}-1 \mid\right.$ $\left.1, \ldots, c_{j}-1, C_{j}+1, \ldots, n\right]$.

By Lemma 3.2, the intersection of the ideals generated by these sets is equal to the ideal generated by the intersection of the sets.

Note, if $B$ is a domain this gives $J$ as an intersection of prime ideals. However it may fail to be a primary decomposition of $J$, as redundancies may arise in the following ways. For example, if $r_{j}>R_{j}-R_{i}$ then every minor containing at least $r_{j}$ rows of $X_{R_{j}}$ must contain at least $r_{j}-\left(R_{j}-R_{i}\right)$ rows of $X_{R_{i}}$; now if $r_{i} \leq r_{j}-R_{j}+R_{i}$ then the condition imposed by $r_{i}$ is implied by the $r_{j}$ condition and the prime ideal $I_{r_{i}}\left(X_{R_{i}}\right)$ is redundant. Or if $t-\left(m-R_{i}\right) \geq r_{i}$ then every $t$-minor has at least $r_{i}$ rows in $X_{R_{i}}$. Finally there are a few trivial situations: if $r_{i}>R_{i}$ the whole thing is zero; if $R_{i}=R_{j}$ or $r_{i}=r_{j}$ then one condition is obviously redundant. These are the only possible redundancies as the following proposition shows.

Proposition 4.4. Suppose

(1) $R_{1}<\cdots<R_{p}$ and $C_{1}<\cdots<C_{q}$,

(2) $r_{1}<\cdots<r_{p}<t$ and $c_{1}<\cdots<c_{q}<t$,

(3) $0 \leq r_{i} \leq R_{i}$ for each $i$ and $0 \leq c_{j} \leq C_{j}$ for each $j$,

(4) $R_{1}-r_{1}<\cdots<R_{p}-r_{p}<m-t$ and $C_{1}-c_{1}<\cdots<C_{q}-c_{q}<n-t$.

Then the intersection (1) is irredundant.

Proof. First, fix $1 \leq i \leq p$. Consider the $t$-minor

$$
m=\left[1, \ldots, r_{i}-1, R_{i}+1, \ldots, t+R_{i}-r_{i}+1 \mid 1, \ldots, t\right] .
$$

We use $R_{i}-r_{i}<m-t$ to verify $t+R_{i}-r_{i}+1 \leq m$, so this is a permissible $t$-minor in an $m \times n$ matrix. For each $j<i, m$ has exactly $\min \left(r_{i}-1, R_{j}\right)$ rows in $X_{R_{j}}$, and this is $\geq r_{j}$, so $m \in I_{r_{j}}\left(X_{R_{j}}\right)$. For each $j>i$, the number of rows of $m$ in $X_{R_{j}}$ is either $t$, if $t+R_{i}-r_{i}+1 \leq R_{j}$, or else $R_{j}-R_{i}+r_{i}-1$, if $R_{i}+1 \leq R_{j} \leq t+R_{i}-r_{i}+1$. In the first case $t \geq r_{j}$ and in the second case $R_{j}-r_{j}>R_{i}-r_{i}$, so $R_{j}-R_{i}+r_{i}-1 \geq r_{j}$; therefore $m \in I_{r_{j}}\left(X_{R_{j}}\right)$. And clearly $m$ has only $r_{i}-1$ rows in $X_{R_{i}}$. This shows that

$$
m \in I_{t}(X) \cap I_{r_{1}}\left(X_{R_{1}}\right) \cap \cdots \cap I_{r_{i-1}}\left(X_{R_{i-1}}\right) \cap I_{r_{i+1}}\left(X_{R_{i+1}}\right) \cap \cdots \cap I_{r_{p}}\left(X_{R_{p}}\right)
$$

but $m \notin I_{r_{i}}\left(X_{R_{i}}\right)$. Clearly $m \in \bigcap I_{c_{j}}\left(X^{C_{j}}\right)$. So the term $I_{r_{i}}\left(X_{R_{i}}\right)$ is irredundant for each $i$. The same argument shows that each $I_{c_{j}}\left(X^{C_{j}}\right)$ is irredundant.

Finally consider the $(t-1)$-minor

$$
m^{\prime}=[1, \ldots, t-1 \mid 1, \ldots, t-1] .
$$

Since each $r_{i}<t, m^{\prime}$ has at least $r_{i}$ rows in each $X_{R_{i}}$ and similarly at least $c_{j}$ columns in each $X^{C_{j}}$. This shows that the term $I_{t}(X)$ is irredundant.

\section{Minors OF SYMmetriC MATRICES}

Now let $Y=\left(y_{i, j}\right)$ be a generic symmetric $n \times n$ matrix, $y_{i, j}=y_{j, i}$. Fix sequences $R=\left(R_{1}, \ldots, R_{p}\right)$ with $1 \leq R_{1} \leq \cdots \leq R_{p} \leq n$ and $r=\left(r_{1}, \ldots, r_{p}\right)$. Let $Y_{R_{i}}$ be the submatrix consisting of the first $R_{i}$ rows of $Y$. We are interested in the $t$-minors that have at least $r_{i}$ rows in $Y_{R_{i}}$ for each $i$. Note, at this point we allow all minors, not only doset minors. 
This is an author-produced, peer-reviewed version of this article. The final, definitive version of this document can be found online at Communications in Algebra, published by Taylor and Francis. Copyright restrictions may apply. doi: 10.1080/00927872.2015.1027375

DECOMPOSITIONS OF IDEALS OF MINORS MEETING A SUBMATRIX

Theorem 5.1. Let $B$ be a ring and $A=B[Y]$. Let $J=J(Y, t, R, r)$ be the ideal generated by $t$-minors of $Y$ that have at least $r_{i}$ rows contained in $Y_{R_{i}}$ for each $1 \leq i \leq p$. Then

$$
J=I_{t}(Y) \cap I_{r_{1}}\left(Y_{R_{1}}\right) \cap \cdots \cap I_{r_{p}}\left(Y_{R_{p}}\right) .
$$

If $B$ is a domain then each $I_{r_{i}}\left(Y_{R_{i}}\right)$ is a prime ideal.

Proof. First, by [5, Lemma 2.3], every $t$-minor $[a \mid b]$ is a linear combination of doset $t$-minors $[c \mid d]$ with $c \leq a$. Thus we can take $J$ to be generated by the doset $t$-minors meeting the row conditions.

Next, each of the following sets is a doset order ideal in $\Delta^{s}(Y)$ :

(1) The set of doset minors of size $\geq t$ is the doset order ideal generated by $[n-t+$ $1, \ldots, n \mid n-t+1, \ldots, n]$.

(2) The set of doset $\left(\geq r_{i}\right)$-minors of $Y_{R_{i}}$ is the doset order ideal generated by $\left[R_{i}-r_{i}+\right.$ $\left.1, \ldots, R_{i} \mid n-r_{i}+1, \ldots, n\right]$. If $[a \mid b] \leq_{1}\left[R_{i}-r_{i}+1, \ldots, R_{i} \mid n-r_{i}+1, \ldots, n\right]$ then $[a \mid b]$ involves at least $r_{i}$ rows of $Y_{R_{i}}$; by Laplace expansion and [5, Lemma 2.3], $[a \mid b]$ is a linear combination of doset $r_{i}$-minors of $Y_{R_{i}}$.

This shows that $J$ is the indicated intersection.

The set of doset $(\geq t)$-minors of $Y$ is cogenerated by $[1, \ldots, t-1 \mid 1, \ldots, t-1]$. The set of $\operatorname{doset}\left(\geq r_{i}\right)$-minors of $Y_{R_{i}}$ is cogenerated by $m=\left[1, \ldots, r_{i}-1, R_{i}+1, \ldots, n \mid 1, \ldots, r_{i}-1, R_{i}+\right.$ $1, \ldots, n]\left[5\right.$, Remark 2.5(c)]. Indeed, $[a \mid b] \nsupseteq_{1} m$ if and only if $a \nsupseteq\left(1, \ldots, r_{i}-1, R_{i}+1, \ldots, n\right)$, if and only if $|a| \geq r_{i}$ and $a_{r_{i}} \leq R_{i}$; so $[a \mid b]$ involves at most $r_{i}$ rows of $Y_{R_{i}}$. This shows that each of the ideals being intersected is cogenerated by a single doset element. Therefore if $B$ is a domain then each of them is a prime ideal by Proposition 3.7.

If $B$ is a domain then once again this writes $J$ as an intersection of prime ideals, but as before it may fail to be a primary decomposition because of redundancy.

Proposition 5.2. Suppose

(1) $R_{1}<\cdots<R_{p}$,

(2) $r_{1}<\cdots<r_{p}<t$,

(3) $0 \leq r_{i} \leq R_{i}$ for each $i$,

(4) $R_{1}-r_{1}<\cdots<R_{p}-r_{p}<n-t$.

Then the intersection (2) is irredundant.

The proof is the same as before.

\section{Pfaffians}

Let $Z=\left(z_{i, j}\right)$ be an $n \times n$ generic skew-symmetric matrix, so that $z_{i, j}=-z_{j, i}$ and $z_{i, i}=0$, and let $A=B[Z]=B\left[\left\{z_{i, j}\right\}\right]$. The Pfaffian of $Z$, denoted $\operatorname{Pf}(Z)$, is a certain polynomial in the entries of $Z$, with the property that $\operatorname{Pf}(Z)^{2}=\operatorname{det}(Z)$. When $n$ is odd, $\operatorname{Pf}(Z)=\operatorname{det}(Z)=0$; for $n=2,4$ we have

$$
\operatorname{Pf}\left(\begin{array}{cc}
0 & a \\
-a & 0
\end{array}\right)=a, \quad \operatorname{Pf}\left(\begin{array}{cccc}
0 & a & b & c \\
-a & 0 & d & e \\
-b & -d & 0 & f \\
-c & -e & -f & 0
\end{array}\right)=a f-b e+c d
$$

In general, for $n$ even,

$$
\operatorname{Pf}(Z)=\sum \operatorname{sgn}(\sigma) z_{\sigma(1), \sigma(2)} \cdots z_{\sigma(n-1), \sigma(n)},
$$


where the sum is over all permutations $\sigma \in S_{n}$ such that $\sigma(2 i-1)<\sigma(2 i)$ for all $i$ and $\sigma(1)<\sigma(3)<\cdots<\sigma(2 n-1)$. Equivalently, the sum is over all unordered partitions of $\{1, \ldots, 2 n\}$ into pairs; the restrictions on $\sigma$ simply amount to choosing one representative ordering for each partition. There is a Laplace-like expansion: for each $j, 1 \leq j \leq n$,

$$
\operatorname{Pf}(Z)=\sum_{i<j}(-1)^{i+j+1} z_{i, j} \operatorname{Pf}\left(Z^{i, j}\right)+\sum_{i>j}(-1)^{i+j} z_{i, j} \operatorname{Pf}\left(Z^{i, j}\right),
$$

where $Z^{i, j}$ is the matrix obtained by deleting the $i$ th and $j$ th rows and columns of $Z$. See $[4,6,7]$.

A $t$-Pfaffian of $Z$ is given by a list of $t$ rows and the same columns; we write briefly $\left[a_{1}, \ldots, a_{t}\right]$, where $1 \leq a_{1}<\cdots<a_{t} \leq n$, for the Pfaffian of the skew-symmetric submatrix given by the rows $a_{1}, \ldots, a_{t}$ and the same columns. Of course this is zero if $t$ is odd.

The ideal generated by the size $t$ Pfaffians of $Z$ is denoted $P_{t}(Z)$. If $n$ is odd then $P_{n-1}(Z)$ is a prime ideal of height 3 . More generally, $P_{2 p}(Z)$ is a prime ideal of height $\mu(p, n)=(n-2 p+1)(n-2 p+2) / 2$, see $[7]$.

We are interested in the ideal generated by the subset of Pfaffians with at least $r_{1}$ rows in the first $R_{1}$ rows of $Z$, at least $r_{2}$ rows in the first $R_{2}$ rows of $Z$, and so on; the row condition implies that these Pfaffians meet the corresponding column conditions as well, i.e., at least $r_{1}$ columns in the first $R_{1}$ columns of $Z$, and so on.

Fix a sequence $1 \leq R_{1} \leq \cdots \leq R_{p} \leq n, R=\left(R_{1}, \ldots, R_{p}\right)$, and another sequence $r=\left(r_{1}, \ldots, r_{p}\right)$ of the same length. Let $Z_{R_{i}}$ be the submatrix of $Z$ consisting of the first $R_{i}$ rows and let $Z_{R_{i}}^{R_{i}}$ be the $R_{i} \times R_{i}$ submatrix of $Z$ in the upper left corner, consisting of the first $R_{i}$ rows and the first $R_{i}$ columns. We will also need, for each $R_{i}+1 \leq k \leq n$, the $\left(R_{i}+1\right) \times\left(R_{i}+1\right)$ submatrix given by the first $R_{i}$ rows and columns plus the $k$ th row and column, that is, the set of rows (and columns) corresponding to the set $\left\{1, \ldots, R_{i}, k\right\}$. Recall the common notation $\left[R_{i}\right]=\left\{1, \ldots, R_{i}\right\}$, so we may write $\left[R_{i}\right] \cup\{k\}$ for the set we want. To simplify notation, we write $Z\left(\left[R_{i}\right]\right)$ for $Z_{R_{i}}^{R_{i}}$ and we write $Z\left(\left[R_{i}\right] \cup\{k\}\right)$ for the $\left(R_{i}+1\right) \times\left(R_{i}+1\right)$ skew-symmetric submatrix of $Z$ given by the rows (and columns) corresponding to the set $\left\{1, \ldots, R_{i}, k\right\}$. Since confusion seems unlikely we will drop the brackets and braces and simply write $Z\left(R_{i}\right)$ and $Z\left(R_{i} \cup k\right)$. Thus for example

$$
Z(3 \cup 5)=\left(\begin{array}{cccc}
0 & z_{1,2} & z_{1,3} & z_{1,5} \\
-z_{1,2} & 0 & z_{2,3} & z_{2,5} \\
-z_{1,3} & -z_{2,3} & 0 & z_{3,5} \\
-z_{1,5} & -z_{2,5} & -z_{3,5} & 0
\end{array}\right),
$$

with rows and columns given by the set $3 \cup 5=[3] \cup\{5\}=\{1,2,3,5\}$.

Theorem 6.1. Let $B$ be a ring and $A=B[Z]$. Let $J=J(Z, 2 t, R, r)$ be the ideal generated by $2 t$-Pfaffians of $Z$ that have at least $r_{i}$ rows in $Z_{R_{i}}$ for $1 \leq i \leq p$. For each $i$, if $r_{i}$ is even, let $J_{i}=P_{r_{i}}\left(Z\left(R_{i}\right)\right)$, and if $r_{i}$ is odd, let $J_{i}=\sum_{k=R_{i}+1}^{n} P_{r_{i}+1}\left(Z\left(R_{i} \cup k\right)\right)$. Then

$$
J=P_{2 t}(Z) \cap J_{1} \cap \cdots \cap J_{p} .
$$

If $B$ is a domain then $P_{2 t}(Z)$ is prime and each $J_{i}$ is a prime ideal.

Proof. Each of the following is an order ideal in $\Pi(Z)$ :

(1) The set of $2 t$-Pfaffians is the order ideal generated by $[n-2 t+1, \ldots, n]$. 
(2) The set of Pfaffians (of all sizes) with at least $r_{i}$ rows contained in $Z_{R_{i}}$. If $r_{i}$ is even, this is the order ideal generated by $\left[R_{i}-r_{i}+1, \ldots, R_{i}\right]$. If $r_{i}$ is odd, this is the order ideal generated by $\left[R_{i}-r_{i}+1, \ldots, R_{i}, n\right]$.

So, by Lemma $3.2, J$ is equal to the intersection of the ideals $P_{2 t}(Z)$ and, for each $i$, the ideal generated by the Pfaffians (of any size) having at least $r_{i}$ rows in $Z_{R_{i}}$.

If $r_{i}$ is even then the ideal generated by Pfaffians with at least $r_{i}$ rows in $Z_{R_{i}}$ is $P_{r_{i}}\left(Z\left(R_{i}\right)\right)$. Indeed, if $P$ is any Pfaffian with at least $r_{i}$ rows in $Z_{R_{i}}$ then $P$ can be expanded as a combination of $r_{i}$-Pfaffians involving those rows.

If $r_{i}$ is odd and $P$ is any Pfaffian with at least $r_{i}$ rows in $Z_{R_{i}}$, then either $P$ actually has at least $r_{i}+1$ rows in $Z_{R_{i}}$ or else $P$ involves at least one more row, say the $k$ th row, with $k>R_{i}$. Either way, $P$ can be expanded as a combination of $\left(r_{i}+1\right)$-Pfaffians in $Z\left(R_{i} \cup k\right)$. So $P$ lies in the sum given in the statement. Conversely, every Pfaffian generator of the sum in the statement must have at least $r_{i}$ rows in $Z_{R_{i}}$.

Now suppose $B$ is a domain. The set of $(\geq 2 t)$-Pfaffians of $Z$ is cogenerated by $[1, \ldots, 2 t-$ 2]. This shows $P_{2 t}(Z)$ is prime. (Of course $P_{2 t}(Z)$ is already well-known to be prime.)

To see that each $J_{i}$ is prime, note that the order ideal of Pfaffians generating $J_{i}$ is cogenerated by either $m=\left[1, \ldots, r_{i}-1, R_{i}+1, \ldots, n\right]$ or $m^{\prime}=\left[1, \ldots, r_{i}-1, R_{i}+1, \ldots, n-1\right]$, whichever has even length (regardless of whether $r_{i}$ is even or odd). Let us verify this. For simplicity, suppose that $m$ has even length. We must show that $\alpha \nsupseteq m$ if and only if $\alpha$ has at least $r_{i}$ rows in $Z_{R_{i}}$, equivalently $\alpha \geq m$ if and only if $\alpha$ has $r_{i}-1$ or fewer rows in $Z_{R_{i}}$; note that this is the criterion whether $r_{i}$ is even or odd. Now $\alpha \geq m$ if and only if $|\alpha| \leq r_{i}-1$ or $|\alpha| \geq r_{i}$ and $\alpha_{r_{i}} \geq R_{i}+1$. The forward direction is obvious; conversely, under these conditions, $\alpha_{r_{i}+t} \geq \alpha_{r_{i}}+t \geq R_{i}+1+t=m_{r_{i}+t}$ for all $0 \leq t \leq|\alpha|-r_{i}$, so each entry of $\alpha$ is at least as great as the corresponding entry of $m$; and in particular since every entry of $\alpha$ is at most $n,|\alpha| \leq|m|$. This shows that $\alpha \geq m$. So indeed $\alpha \geq m$ if and only if $\alpha$ has $r_{i}-1$ or fewer rows in $Z_{R_{i}}$.

The argument in case $\left|m^{\prime}\right|$ is even is similar. Note that $m^{\prime}$ is as long as possible for a member of $\Pi(Z)$ with $R_{i}+1$ in the $r_{i}$ position; so if $\alpha_{r_{i}} \geq R_{i}+1$ then $|\alpha| \leq\left|m^{\prime}\right|$.

Once again this writes $J$ as a possibly redundant intersection of prime ideals, if $B$ is a domain.

Proposition 6.2. Suppose

(1) $R_{1}<\cdots<R_{p}$,

(2) $r_{1}<\cdots<r_{p}<2 t$

(3) $0 \leq r_{i} \leq R_{i}$ for each $i$,

(4) $R_{1}-r_{1}<\cdots<R_{p}-r_{p}<n-2 t$.

Then the intersection (3) is irredundant.

The proof is the same as before.

Corollary 6.3. Let $Z=\left(z_{i, j}\right)$ be a generic skew-symmetric matrix, let $0<p<q$, and fix $A=B[Z]$ with degree $\operatorname{deg} z_{i, j}=2 p$ if $i, j \leq R$, $\operatorname{deg} z_{i, j}=p+q$ if $i \leq R<j$, and $\operatorname{deg} z_{i, j}=2 q$ if $i, j>R$. Fix $t$ and $d$. Let $r=\left\lceil\frac{2 t q-d}{q-p}\right\rceil$. Then $P_{2 t}(Z)_{\leq d}=P_{2 t}(Z) \cap I_{r}$ where $I_{r}$ is the ideal generated by Pfaffians with at least $r$ rows in $Z_{R}$. If $r$ is even, $I_{r}=P_{r}(Z(R))$. If $r$ is odd, $I_{r}=\sum_{k=R+1}^{n} P_{r+1}(Z(R \cup k))$ where $Z(R \cup k)$ is the $(R+1) \times R+1$ skew-symmetric submatrix of $Z$ given by the rows (and columns) corresponding to the set $\{1, \ldots, R, k\}$. If $B$ is a domain then $P_{2 t}(Z)$ and $I_{r}$ are prime. 
Indeed, a $2 t$-Pfaffian $P$ with $r$ rows in $Z_{R}$ has degree $p r+q(2 t-r)$; the value of $r$ in the statement is the least integral solution to $\operatorname{deg} P \leq d$.

\section{REFERENCES}

1. J. F. Andrade and A. Simis, On ideals of minors fixing a submatrix, J. Algebra 102 (1986), no. 1, 246-259. MR 853243 (87j:13028)

2. Cornel Baetica, Combinatorics of determinantal ideals, Nova Science Publishers Inc., Hauppauge, NY, 2006. MR 2298637 (2008h:13023)

3. Winfried Bruns and Udo Vetter, Determinantal rings, Lecture Notes in Mathematics, vol. 1327, SpringerVerlag, Berlin, 1988. MR 953963 (89i:13001)

4. David A. Buchsbaum and David Eisenbud, Algebra structures for finite free resolutions, and some structure theorems for ideals of codimension 3, Amer. J. Math. 99 (1977), no. 3, 447-485. MR 0453723 (56 \#11983)

5. Aldo Conca, Gröbner bases of ideals of minors of a symmetric matrix, J. Algebra 166 (1994), no. 2, 406-421. MR 1279266 (95g:13012)

6. P. Heymans, Pfaffians and skew-symmetric matrices, Proc. London Math. Soc. (3) 19 (1969), 730-768. MR 0257105 (41 \#1759)

7. Tadeusz Józefiak and Piotr Pragacz, Ideals generated by Pfaffians, J. Algebra 61 (1979), no. 1, 189-198. MR 554859 (81e:13005)

8. Ronald E. Kutz, Cohen-Macaulay rings and ideal theory in rings of invariants of algebraic groups, Trans. Amer. Math. Soc. 194 (1974), 115-129. MR 0352082 (50 \#4570)

9. Fatemeh Mohammadi, Prime splittings of determinantal ideals, arXiv:1208.2930 [math.AC], Aug 2012.

10. Zachariah C. Teitler, Multiplier ideals of general line arrangements in $\mathbb{C}^{3}$, Comm. Algebra 35 (2007), no. $6,1902-1913$.

11. __ On the intersection of the curves through a set of points in $\mathbb{P}^{2}$, JPAA 209 (2007), no. 2, 571-581.

Kent M. Neuerburg, Department of Mathematics, Slu 10687, Hamond, LA 70401, USA

E-mail address: kneuerburg@selu.edu

Zach Teitler, Department of Mathematics, Boise State University, 1910 University Drive, BoIse, ID 83706, USA

E-mail address: zteitler@boisestate.edu 